\title{
Multiagent Chemopreventive Agent Combinations
}

\author{
Dean E. Brenner, M.D.* \\ University of Michigan M edical Center
}

\begin{abstract}
Cancer chemoprevention is a new discipline whose foundation rests upon epidemiologic evidence suggesting that dietary components such as beta-carotene, vitamin E, calcium and selenium may be inhibitors of carcinogenesis. $O$ ver the last decade, as molecular and biochemical mechanisms of the carcinogenesis process have been elucidated, the rationale of combining chemopreventive agents to target multiple pathways has strengthened. The process of identifying potential synergistic combinations of chemoprevention agents should be based upon a systematic process of preclinical development in vitro followed by testing in animal models of carcinogenesis. Surrogates of anticarcinogenesis effects might include biochemical, molecular and pathologic assessment of tissue from animal carcinogenesis models. If evidence of chemopreventive effect is found in animal models, systematic studies in humans are indicated. These studies should include a careful Phase I trial to describe optimal chemoprevention doses for all agents being tested in combination followed by Phase II trials to assess efficacy upon carcinogenesis biological and pathological surrogates. J. Cell. Biochem. Suppl. 34:121-124, 2000. ๑ 2000 Wiley-Liss, Inc.
\end{abstract}

Key words: chemoprevention; biomarkers; drug development

\section{INTRO DUCTION}

Combinations of cytotoxic agents with nonoverlapping toxicity while enhancing therapeutic effect has resulted in important treatment advances for patients with lymphomas, childhood tumors, leukemias, and germinal cell neoplasms [DeVita, 1997]. I nitial experimental data in vitro and in vivo demonstrated enhanced cytotoxic effects for combinations of cytotoxic agents when compared to the same agents given alone. Combinations of drugs selected had in vitro evidence of additive or synergistic efficacy, clinical evidence of single agent activity, and toxicity profiles that differed sufficiently to allow for some, if not complete overlap [DeVita, 1997]. The concept of multiagent cytotoxic therapeutics for cancer treatment became firmly established in the 1960s with evidence of enhanced survival efficacy in childhood leukemias and Hodgkins disease after combination cytotoxic chemotherapy when compared to single agent chemotherapy [DeVita, 1997].

Can a similar rationale support the use of combinations of chemopreventive agents to en-

*Correspondence to: Dean E. Brenner, M.D., 3-215 Cancer and Geriatrics Center, University of Michigan Medical Center, 1500 Medical Center Drive, Ann Arbor, MI 48109-0934. Email: dbrenner@umich.edu

Received February 3, 1999; Accepted March 8,1999 hance anticarcinogenesis effect? If so, how might the lessons learned from experience with combination cytotoxic agents be applied to drug or micronutrient treatments aimed at preventing cancer ? I n the past, combinations of chemopreventive agents have been based primarily upon epidemiologic criteria. How should future chemopreventive agent combinations be developed? What existing mechanistic, preclinical and clinical efficacy data are necessary prior to initiation of clinical trials? How should efficacy assessment of combinations of chemopreventive agents proceed?

This short discussion addresses these issues and suggests experimental paradigms that may be predinically and clinically tested.

\section{CHEMO PREVENTIVE AGENT CO MBINATIONS USED IN LARGE RISK REDUCTION TRIALS}

Cancer chemoprevention is a new discipline whose foundation rests upon epidemi ologic evidence suggesting that dietary components including vitamins and micronutrients such as beta-carotene, vitamin E, calcium, and selenium may be inhibitors of carcinogenesis. $\mathrm{Re}$ cently publ ished risk reduction trials for chemoprevention of lung, esophageal, and gastric cancers have empl oyed combinations of chemopreventive agents [Blot et al., 1993; Heinonen and Albanes, 1994; Omenn et al., 1996]. The 
micronutrient agents used in these large trials were chosen primarily, but not solely, on the basis of epidemiologic data. The CARET investigators carefully reviewed evidence of single agent micronutrient efficacy in animal carcinogenesis models [Peto et al., 1981]. They also reviewed data in banked blood samples and human epidemiologic data prior to initiation of their trial of beta-carotene and retinol for lung cancer risk reduction trial in smokers who had stopped smoking. Despite this careful preliminary analysis, both this trial and a similar trial in Finland [Heinonen and Albanes, 1994] failed to demonstrate a reduction in lung cancer incidence in the target population.

Blot et al. [1993] justified testing the chemopreventive activities of selenium, beta-carotene, al pha-tocopherol, zinc, riboflavin and niacin in Chinese subjects on the basis of epidemiology, blood samples and experimental animal dietary supplemental data. Specific nutritional supplementation studies in combination of the proposed agents were not performed. The multi-agent, factorial design made it difficult to discern the relative contributions of the three chemopreventive agents administered to the Chinese subjects.

In hind-sight, one is tempted to argue that the failure of the two large prospective risk reduction trials of beta-carotene in combination with other micronutrients as a lung cancer preventive may be due to the lack of preliminary mechanism-based data, of strong synergism data in animal carcinogenesis models, and of careful dose-response-toxicity data preclinically and clinically. However, these large studies provide a wealth of exploitable insights into the design, cost and execution of multi-agent chemoprevention trials for the future.

\section{MECHANISTIC RATIO NALE FO R MU LTIAGENT CHEMO PREVENTION TRIALS}

Although the precise biological mechanisms of cellular carcinogenesis are incomplete, a number of specific mechanisms are well documented to be procarcinogenic and may be explored in in vitro and in vivo models. Table 1 lists potential chemopreventive mechanisms. These mechanisms have been categorized by the $\mathrm{N}$ ational Cancer I nstitute into three broad categories: 1. cancer blocking , 2. antioxidant, and 3. antiproliferation/antiprogression mechanisms. Enzymatic screening assays and in vitro methods are used to assess agent-activity profiles in
TAB LE I. Measurable and Targetable Chemopreventive Mechanisms*

Cancer-Blocking Activities
Prevent carcinogen binding to DNA
Glutathione reduction
Phasell enzyme induction
Antioxidant Activities
Microsomal enzyme inhibition
Free radical/superoxide inhibition
Arachidonic acid metabolism inhibition
Antiproliferation/Antiprogression/Apoptosis I nduc-
tion
Signal transduction modulation
Calmodulin inhibition
Polyamine synthesis inhibition
Polymerase inhibition/induction
Apoptosis induction
Differentiation enhancement
Modulation of growth factor and hormonal
activity
Angiogenesis inhibition
Arachidonic acid metabolism inhibition
Inhibition of basement membrane degradation
Prevention of oncogene activation
Stimulation of immune response
Promotion of intracellular communication

*Adapted from Steele et al. [1998] with permission.

each of these categories and contribute to an overall assessment of the likelihood of anticarcinogenesis effect in humans [Steele et al., 1998]. The rapid pace of discovery of mechanisms of cellular signal transduction, growth and apoptosis control will rapidly obsolesce this table.

Combinations of chemoprevention agents are being designed to exploit multiple mechanisms listed. For example, in animal models, all-trans$\mathrm{N}$-(4-hydroxyphenyl)retinamide (antiproliferative and apoptosis induction) and tamoxifen (modulation of growth factor and hormonal activity-antiproliferation via different mechanisms) appear to havesynergistic chemopreventive activity to prevent tumor recurrence in $\mathrm{N}$-methyl-N-nitrosourea treated rats [Ratko et al., 1989]. The combination of DFMO (polyamine inhibition-antiproliferation) and piroxicam (non-steroidal antiinflammatory inhibitor) synergistically reduces the number, size, and incidence of colon tumors in azoxymethane induced col onic neoplasias [Rao et al., 1991]. Both of these models are being clinically tested in Phase I and II chemoprevention trials. Other examples of proposed chemopreventive agent combinations include $\mathrm{N}$-acetyl-1-cysteine (antioxidant) and oltipraz (antioxidant). The ratio- 
nale of combining two antioxidants rests in dual mechanisms of enhancement of intracellular glutathione. Oltipraz enhances cellular synthesis of glutathione while $\mathrm{N}$-acetyl-I-cysteine provides cysteine for glutathione synthesis.

Selective enzymeinhibitors should allow more mechanism specific and targeting chemoprevention combinations. For example, sel ectiveestrogen receptor inhibitors and nonsteroidal antiinflammatory agents have been approved for non-cancer preventive indications. In a secondary endpoints analysis, raloxafene, a selective estrogen-receptor inhibitor, reduces the risk of breast cancer in women treated for control of osteoporosis [Cummings et al., 1998]. Aspirin, a known cardiovascular preventive reduces the risk of colorectal cancer in large epidemiologic cohorts. At least two mor eselective cycl ooxygenase-2 inhibitors are likely to enter the market within the next year. Preliminary data in animal models supports the high potency of cyclooxygenase-2 inhibitors as colorectal cancer chemopreventives [Kawamori et al., 1998]. With specific protein kinase $C$ inhibitors, antisense oligonucleotides specific for signal transduction genes under development, the likelihood for pathway specific anticarcinogenesis therapeutics is high. Combinations of pathway specific agents will permit probing of cellular proliferative and apoptotic control pathways clinically. Such a mechanistic approach to combined chemopreventive agent therapeutics is an attractive alternative to current methods of combination of chemopreventive agents.

\section{PRELIMINARY DATA REQ UIRED PRIOR TO COMBINATIO N CHEMO PREVENTIVE TRIALS IN HUMANS}

Prior to human clinical trials of any new drug, Food and DrugAdministration standards for each drug to be tested in combination for safety should be met. This includes the determination of pharmacokinetic parameters in acute and subchronic toxicity testing, reproductive studies, and genotoxicity evaluation in animal model systems. Proposed chemopreventive agent combinations should al so be evaluated in at least one preclinical animal model system for pharmacokinetic, toxicity, or enzyme interactions [Steele et al., 1998]. Rao et al. [1991] studied the chemopreventive action of $40 \%$ and $80 \%$ maximum tolerated doses levels of piroxicam, difluoromethylornithine (DFMO), 16- $\alpha$ fluoro-5-androsten-17-one (DHEA analogue), and ellagic acid in two and three drug combinations. The two dose levels chosen were orally administered to $\mathrm{F} 344$ rats given azoxymethane as a colonic carcinogen. The DFMO-piroxicam combination had enhanced anticarcinogenesis effect over either al one. Although isobolic analysis to define synergism was not possible in this study design, this experiment provided important data that support further clinical development of the DFMO-piroxicam combination.

\section{APPRO ACH TO HUMAN CLINICAL TRIALS}

The dilemma of defining appropriate endpoints for early stage chemoprevention compounds the problem of assessing multi-agent chemopreventive combinations. To date, pathologic surrogate endpoints remain the preferred approach to the preliminary assessment of chemopreventive efficacy. Surrogates such as coIonic polyps, actinickeratoses, cervical intraepithelial neoplasia may be useful indicators of interventional drug effect.

Phase I trials of chemopreventive agent combinations may consist of escalating doses of each agent in groups of 3 to 6 subjects, treated for a short period of time (14 to 28 days). In addition to toxicity assessment, endpoints may consist of drug effect endpoints in tissues such as colonic mucosal prostaglandin content and polyamine content for a DFMO-piroxicam Phase I combination trial. As pathway-enzyme specific inhibitors become available, the target enzymes and products in morphologically normal tissue and in pathologic surrogates may be assayed for drug effect. A successful chemopreventive agent combination combines evidence of target-specific efficacy with acceptable clinical toxicity. Maximum tolerable doses need not be defined. Rather, optimal doses that can reproducibly alter a known biochemical target in a large proportion of healthy human subjects $(>67 \%)$ are defined in the Phase I trial. Although time consuming, these Phase I trials have great importance in defining the best suitable dose and schedule for a given combination. Since the goal of chemopreventive interventions is to protect cells from carcinogenic stress or slow or stem the carcinogenesis process, definition of maximum tolerable doses is not essential. Rather, Phase I trials of chemopreventive agent combinations should identify safe and effective doses the alter a carcinogenesis endpoint for each agent in the combination. 
OncePhasel trials identify effective combination doses, Phase IIa and II b clinical trials to assess chemopreventive effect upon surrogate endpoints may proceed. Phase II trials provide sufficient data to determine whether the anticarcinogenesis effect of a combination of chemoprevention agents is sufficient to warrant a large risk reduction trial. Phase II a trials may complete or refine combination dose and schedule by an open label, randomized design with a tissue surrogate or biological surrogate endpoint. Phase II b trials are randomized, blinded trials of intermediate treatment lengths (6 months to a year) with tissue surrogate endpoints, biological surrogate endpoints or both. The design of these trials provide sufficient data and statistical power to define a combination's potential as cancer risk reduction treatment and whether a risk reduction trial should be undertaken.

\section{SUMMARY AND CONCLUSIONS}

Given the current state of the art in chemopreventive agent development, combining agents to obtaining sufficient preliminary data in humans to justify testing in large, risk reduction trials is a challenging, high risk but high gain endeavor. Prior experience in large cohort, multinutrient risk reduction chemoprevention trials suggests that more careful preliminary efforts in smaller cohorts using biological surrogates of cellular transformation or carcinogenesis are indicated. Preliminary data in animal models of chemical or transgenic carcinogenesis provides necessary information regarding dose, frequency, and synergism that may be used to justify clinical development of combination agents. Human Phase I trials to define optimal anticarcinogenesis combination chemopreventive doses upon a surrogate endpoint are necessary prior to efficacy assessments in Phase II or III. Exploiting anticarcinogenic targeting agents in a sequence aimed at reducing cellular proliferative signals while enhancing apoptotic function may be an optimal future approach to the design and testing of chemopreventiveagent combinations.

\section{REFERENCES}

Blot W, Li J , Taylor P, Guo W, Dawsey G-Q, Wang C, Zheng S-F, Gail M, Li T, Yu Y, Liu B, Tangrea J, Sun Y, Liu F, Fraumeni J , You-Hui Z, and Li B. 1993. Nutrition intervention trials in Linxian, China: supplementation with specific vitamin/mineral combinations, cancer incidence and dis-ease specific mortality in the general population. $\mathrm{J} \mathrm{NCl}$ 85:1483-92.

Cummings S, Norton L, Eckert S, Grady D, Cauley J , Knickerbocker R, Black D, Nickelson T, Glusman J , Krueger K.1998. Raloxifene reduces the risk of breast cancer and may decrease the risk of endometrial cancer postmenopausal women. Two year findings from the multiple outcomes of raloxifene evlauation (MORE) trial. Proc of ASCO 17:2a.

DeVita V. 1997. Principles of cancer management: chemotherapy. In DeVita V, Hellman S, Rosenberg S, editors. Cancer: Principles \& Practice of Oncology. Philadel phia: Lippincott-Raven, p 333-347.

Heinonen O, Albanes D. 1994. The effect of vitamin E and beta-carotene on the incidence of lung cancer and other cancers in male smokers. N Engl J Med 330:1029-1035.

Kawamori T, Rao C, Seibert K, Reddy B. 1998. Chemopreventive effect of celecoxib, a specific cyclooxygenase-2 inhibitor on colon carcinogenesis. Cancer Res 58:409412.

Omenn G, Goodman G, Thornquist M, Balmes J , Cullen M, Glass A K, J P, Meyskens F, Valanis B, Williams J , Barnhart S, Hamar S. 1996. Effects of a combination of beta carotene and vitamin A on lung cancer and cardiovascular disease. N Engl J Med 334:1150-1155.

Peto R, Doll R, Buckley J, Sporn M. 1981. Can dietary beta-carotene materially reduce human cancer rates? Nature 290:1-8.

Rao CV, Tokumo K, Rigotty J , Zang E, Kell off G, Reddy BS. 1991. Chemoprevention of colon carcinogenesis by dietary administration of piroxicam, alpha-difluoromethyIornithine, 16 alpha-fluoro-5-androsten- 17-one, and ellagic acid individually and in combination. Cancer Res 51:4528-34.

Ratko T, Detrisac C, Dinger N, Thomas C, Kelloff G, Moon R. 1989. Chemopreventive efficacy of combined retinoid and tamoxifen treatment following surgical excision of a primary mammary cancer in female rats. Cancer Res 49:4472-4476.

Steele V, Boone C, Lubet R, Crowell J , Holmes C, Sigman C, Kelloff K. 1998. Preclinical development paradigms for chemopreventives. Heme/Onc Clinics NA 12:943-961. 